\title{
Fault Point Detection with the Bank of Competitive Kalman Filters ${ }^{\star}$
}

\author{
Innokenti Semoushin, Julia Tsyganova, and Maria Kulikova \\ Ulyanovsk State University, 42 L. Tolstoy Str., 432970 Ulyanovsk, Russia \\ \{SemoushinIV, TsyganovaJuV, KulikovaMV\}@ulsu.ru \\ http://staff.ulsu.ru/semousin/
}

\begin{abstract}
The problem of fault point detection in the linear stochastic discrete systems is considered. To solve this problem the algorithm with the finite size of the Bank of competitive Kalman filters is suggested. Theoretical results are confirmed by numerical experiments.
\end{abstract}

\section{Introduction}

The fault point detection problem has been discussed in many papers (see, for example [1, 2 , [3). One of the possible solutions to this problem is to use the Bank of competitive Kalman filters and some hypotheses testing algorithm. However, there is the problem of practical implementation of the Bank due to the increase in the number of Kalman filters in direct proportion with the length of testing interval. This causes large computational expenses.

To avoid this drawback, a new algorithm with the Bank of bounded number of Kalman filters is suggested in this paper. The authors multiple numerical experiments indicate the efficiency of this new algorithm for the fault point detection.

\section{The SPRT for the Problem of Fault Detection}

Consider the problem of detecting whether a stochastic discrete time system has one set of parameters or another.

Let the system be characterized by the following equations:

$$
\begin{gathered}
x_{t+1}=\Phi_{t} x_{t}+B_{t} u_{t}+\Gamma_{t} w_{t} \\
z_{t}=H_{t} x_{t}+v_{t}
\end{gathered}
$$

where $x_{t}$ is the $n$-dimensional state vector, $z_{t}$ is the $m$-dimensional system output, $u_{t}$ is the control input, and $\left\{w_{0}, w_{1}, \ldots\right\}$ and $\left\{v_{1}, v_{2}, \ldots\right\}$ are mutually independent zero-mean Gaussian sequences of independent vectors. Without loss of

\footnotetext{
* This work was supported in part by the Russian Ministry of Education (grant
} No. T02-03.2-3427). 
generality, their covariances $Q$ and $R$ are assumed to be reduced to identity matrices: $Q=I$ and $R=I$. This can be easily done by normalizing input noise in (1) and measurements in (2). The sequences are considered independent of Gaussian initial $x_{0}$ with mean $\bar{x}_{0}$ and $P_{0}$. At any time $t$, we need to be ascertained, by performing a test on the sequence of measurements $Z_{t}=\left\{z_{1}, z_{2}, \ldots, z_{t}\right\}$, which of the following two hypotheses is true.

Hypothesis $\mathcal{H}_{0}$ : $\quad$ system parameters are $\Phi_{t 0}, B_{t 0}, \Gamma_{t 0}$, and $H_{t 0}$.

Hypothesis $\mathcal{H}_{1}$ : $\quad$ system parameters are $\Phi_{t 1}, B_{t 1}, \Gamma_{t 1}$, and $H_{t 1}$.

Consider two Kalman competitive filters designed, correspondingly, under the assumption of hypothesis $\mathcal{H}_{0}$ or $\mathcal{H}_{1}$. Let subscript $i$ denote the hypothesis number, i.e. filter number, then the filter equations are:

\section{Time propagation}

$$
\hat{x}_{t i}^{-}=\Phi_{t i} \hat{x}_{t-1, i}^{+}+B_{t i} u_{t}, \quad P_{t i}^{-}=\Phi_{t i} P_{t-1, i}^{+} \Phi_{t i}^{T}+\Gamma_{t i} \Gamma_{t i}^{T} .
$$

$$
\begin{array}{ll}
\text { Vector measurement update } & \\
K_{t i}=P_{t i}^{-} H_{t i}^{T}\left(H_{t i} P_{t i}^{-} H_{t i}^{T}+I\right)^{-1}, & P_{t i}^{+}=\left(I-K_{t i} H_{t i}\right) P_{t i}^{-} \\
\nu_{t i}=z_{t}-H_{t i} \hat{x}_{t i}^{-}, & \hat{x}_{t i}^{+}=\hat{x}_{t i}^{-}+K_{t i} \nu_{t i} .
\end{array}
$$

Each of the following sequences

$$
\mathrm{N}_{t 0}=\left\{\nu_{10}, \nu_{20}, \ldots, \nu_{t 0}\right\} \text { and } \mathrm{N}_{t 1}=\left\{\nu_{11}, \nu_{21}, \ldots, \nu_{t 1}\right\}
$$

consists of mutually independent entries $\nu_{\tau i}=z_{\tau}-H_{\tau i} \hat{x}_{\tau i}^{-}, \tau=1,2, \ldots, t$, subject to the condition that the corresponding hypothesis, $i=0$ or $i=1$, is true.

The Wald sequential probability ratio test contains the following recursive algorithm to evaluate the likelihood ratio $\lambda_{t}=\ln p\left\{\mathrm{~N}_{t 1} \mid \mathcal{H}_{1}\right\} / p\left\{\mathrm{~N}_{t 0} \mid \mathcal{H}_{0}\right\}$ :

$$
\lambda_{t}=\lambda_{t-1}+\mu_{t}, \quad t \geq 1
$$

where

$$
2 \mu_{t}=\log \operatorname{det} \Sigma_{t 0}-\log \operatorname{det} \Sigma_{t 1}+\nu_{t 0}^{T} \Sigma_{t 0}^{-1} \nu_{t 0}-\nu_{t 1}^{T} \Sigma_{t 1}^{-1} \nu_{t 1}
$$

The mean and variance of sequences (4) for filters $i=0,1$ are given by

$$
E\left\{\nu_{t i}\right\}=0, \quad E\left\{\nu_{t i} \nu_{t i}^{T}\right\}=\Sigma_{t i}=H_{t i}^{T} P_{t i}^{-} H_{t i}+I .
$$

The value of $\lambda_{t}$ is then tested against two threshold levels $A$ and $B$ (where $A>B)$ :

$$
\left\{\begin{array}{l}
\text { if } \lambda_{t} \leq B, \quad \text { the test is terminated with the choice of } \mathcal{H}_{0} . \\
\text { if } \lambda_{t} \geq A, \quad \text { the test is terminated with the choice of } \mathcal{H}_{1} . \\
\text { if } A>\lambda_{t}>B, \text { the test is repeated. }
\end{array}\right.
$$

The thresholds $A$ and $B$ are chosen in the following way. Let $\alpha$ be the probability of choosing $\mathcal{H}_{1}$ when $\mathcal{H}_{0}$ is true, and $\beta$ be the probability of choosing $\mathcal{H}_{0}$ when $\mathcal{H}_{1}$ is true. Then

$$
A=\log \frac{1-\beta}{\alpha} \quad \text { and } \quad B=\log \frac{\beta}{1-\alpha} .
$$


The initial value of the test is $\lambda_{0}=\log \left\{P_{1} / P_{0}\right\}$, where $P_{0}$ and $P_{1}$ are a'priori probabilities of occurrence of hypotheses $\mathcal{H}_{0}$ and $\mathcal{H}_{1}$. If $P_{0}=P_{1}=\frac{1}{2}$ (i.e. hypotheses $\mathcal{H}_{0}$ and $\mathcal{H}_{1}$ are equiprobable), then it is clear that the initial value $\lambda_{0}=0$.

The SPRT requires the computation of likelihood ratio function $\lambda_{t}$ at each moment $t$. There was suggested an efficient algorithm to compute a likelihood function for any competitive filter [4. The basis for this algorithm is the Conventional Kalman Filter with scalar measurement update. The authors obtained two equivalent proofs of the equivalence between two methods of likelihood function evaluation: the vector method with the help of equations (3)-(5) and the scalar method according to the suggested algorithm [4]. The first proof is based on the Information form of Kalman Filter [4], and the second one is algebraic [5]. In the same manner as the Conventional Kalman Filter, one can modify [4] the other data processing algorithms that are described in [6] and based on the covariance factorization: Potter Square Root; Bierman LD-Covariance Factorization; and Carlson Triangular Covariance Square Root.

\section{Competitive Fault Point Detection Using a Bank of Kalman Filters}

In this section, we solve the problem of fault point detection. The fault point is the time instant at which the parameters of system (1), (2) were changed.

Suppose that hypothesis $\mathcal{H}_{0}$ describes the nominal mode of system operation, i.e. the fault-free mode.

\subsection{The Exact Solution to the Problem of Fault Point Detection}

Consider that a ratio test is started at time $t=t_{0}$, but the point of fault occurrence $t_{01} \in\left[t_{0}, t_{N}\right]$, (where $t_{i} \in\left[t_{0}, t_{N}\right]$ is a testing interval) is unknown. Therefore, in addition to the main hypothesis $\mathcal{H}_{0}, N-1$ alternative hypotheses $\mathcal{H}_{j}$ are added instead of one alternative hypothesis $\mathcal{H}_{1}$. Each $\mathcal{H}_{j}$ means that at the moment $t_{j}$, the system parameters are changed from $\left\{\Phi_{t 0}, B_{t 0}, \Gamma_{t 0}\right.$, and $\left.H_{t 0}\right\}$ to $\left\{\Phi_{t 1}, B_{t 1}, \Gamma_{t 1}\right.$, and $\left.H_{t 1}\right\}$.

Taking into account these prior assumptions, we can write down the expression for the likelihood ratio function:

$$
\lambda_{t}^{j 0}=\log \frac{P\left\{\nu_{j 1}^{j}, \ldots, \nu_{t 1}^{j} \mid \mathcal{H}_{j}\right\}}{P\left\{\nu_{j 0}, \ldots, \nu_{t 0} \mid \mathcal{H}_{0}\right\}}=\lambda_{t-1}^{j 0}+\mu_{t}^{j 0}, \quad t \geq 1
$$

where

$$
2 \mu_{t}^{j 0}=\log \operatorname{det} \Sigma_{t 0}-\log \operatorname{det} \Sigma_{t 1}^{j}+\nu_{t 0}^{T} \Sigma_{t 0}^{-1} \nu_{t 0}-\left(\nu_{t 1}^{j}\right)^{T}\left(\Sigma_{t 1}^{j}\right)^{-1} \nu_{t 1}^{j} .
$$

The value $\lambda_{t}^{j 0}$ needs to be calculated at the moment $t=t_{0}+j$, where $t \in$ $\left[t_{0}, t_{N}\right]$. 
In Sect. 2, two Kalman filters were required, one based on the assumption of hypothesis $\mathcal{H}_{0}$ and the other on assumption of $\mathcal{H}_{1}$. In the present case, we need one Kalman filter per hypothesis, that is, $N+1$ filters.

Let us denote the Kalman filter based on $\mathcal{H}_{0}$ by $\mathcal{F}_{0}$ and the Kalman filters based on $\mathcal{H}_{j}$ by $\mathcal{F}_{j}, j=1, \ldots, N$. Then in $(7), \nu_{t 1}^{j}$ is a residual with the covariance $\Sigma_{t 1}^{j}$, obtained from the Kalman filter $\mathcal{F}_{j}$. So we have the Bank of $N+1$ competitive Kalman filters.

The ratio test for system fault point detection is as follows [3]:

(1. If $\forall t<t_{N} \lambda_{t}^{j 0} \leq B$, the test is terminated with the choice of $\mathcal{H}_{0}$ (the system fault point was not detected on the testing interval).

2. If $\exists ! j: \lambda_{t}^{j 0} \geq A$, the test is terminated with the choice of $\mathcal{H}_{j}$ (the system fault point was detected at the moment $t_{0}+j$ ).

3. If $\forall j: B<\lambda_{t}^{j 0} \leq A$, the test is repeated for $t+1$.

4. If $\exists i \neq j: \lambda_{t}^{j 0} \geq A$ and $\lambda_{t}^{i 0} \geq A$, the test is terminated with the choice of "leading" hypothesis $\mathcal{H}_{l}$, for which the value of likelihood function is maximum, that is $\lambda_{t}^{l 0}=\max \left\{\lambda_{t}^{j 0}, \lambda_{t}^{i 0}\right\}$ (the system fault point was detected at the moment $t_{0}+l$ ).

5. If $\forall i, j \lambda_{t}^{j 0} \leq B$ and $B<\lambda_{t}^{i 0}<A$, the hypothesis $\mathcal{H}_{j}$ is excluded from the set of the considered hypothesis and the test is repeated for $t+1$.

Now we can describe the testing algorithm:

The test is performed on the testing interval $\left[t_{0}, t_{N}\right]$; from the beginning of the test at each subsequent moment $t=j$, the Kalman filter $\mathcal{F}_{j}$ based on $\mathcal{H}_{j}$ is included into the contest of filters (into the Bank of Kalman filters).

Thus, after processing $N$ measurements $N+1$ filters are added to the Bank, moreover each filter $\mathcal{F}_{j}, j=1, \ldots, N$ matches likelihood against filter $\mathcal{F}_{0}$.

If all hypotheses $\mathcal{H}_{j}, j=0, \ldots, N$, are equiprobable, then the initial value of each likelihood ratio function $\lambda_{t}^{j 0}$ equals zero. When a'priori probabilities of all hypotheses are given, the initial condition for the functioning of filter $\mathcal{F}_{j}$ is the value $\lambda_{0}^{j 0}=\log \left\{P_{j} / P_{0}\right\}$, where $P_{j}$ is an a'priori probability of hypothesis $\mathcal{H}_{j}$.

So, the value $\lambda_{t}^{j 0}$ begins to change at Step $j$ of the test at the moment of filter $\mathcal{F}_{j}$ connection. For all $t<t_{0}+j, \lambda_{t}^{j 0}=0$, that corresponds with the condition that the filter is disconnected.

It is clear that according to the suggested algorithm, the number of competitive filters grows at each step of the test. This, in turn, leads to the growth of necessary calculations of likelihood ratio function at each step of the test. The realization of such algorithm in an ideal case will require unlimited computational resources (machine time and an enormous memory size for the data storing).

Therefore in spite of the simplicity of the suggested algorithm and the guaranteed solution to the problem, there is the essential drawback that the algorithm is not practically realizable for the solving of real life problems because of the unbounded growth of computations at each step of the test. 
In the next section, we solve the problem of elimination of such obvious drawback of the obtained solution, i.e. the unbounded growth of the number of competitive filters.

\subsection{The New Algorithm of Fault Point Detection with the Bank of Finite Number of Filters}

From the previous topic, there follows the necessity to find the solution to the problem of Fault Point Detection with the Bank of finite Number of Kalman filters. In other words, the problem to evaluate the necessary number of competitive Kalman filters is posed.

We need to determine the average sample numbers required for decision making between the two hypotheses $\mathcal{H}_{1}$ and $\mathcal{H}_{0}$. We first need to know the average values of the increment in the probability ratio $\lambda_{t}$ at each stage in the test.

In other words, we need to obtain expressions for

$$
\begin{aligned}
& \mu_{1}=E\left\{\log \left(\operatorname{det} \Sigma_{t 0}\right)^{1 / 2}-\log \left(\operatorname{det} \Sigma_{t 1}\right)^{1 / 2}+\frac{1}{2}\left\{\nu_{t 0}^{T} \Sigma_{t 0}^{-1} \nu_{t 0}-\nu_{t 1}^{T} \Sigma_{t 1}^{-1} \nu_{t 1}\right\} \mid \mathcal{H}_{1}\right\} \\
& \mu_{0}=E\left\{\log \left(\operatorname{det} \Sigma_{t 0}\right)^{1 / 2}-\log \left(\operatorname{det} \Sigma_{t 1}\right)^{1 / 2}+\frac{1}{2}\left\{\nu_{t 0}^{T} \Sigma_{t 0}^{-1} \nu_{t 0}-\nu_{t 1}^{T} \Sigma_{t 1}^{-1} \nu_{t 1}\right\} \mid \mathcal{H}_{0}\right\}
\end{aligned}
$$

at time $t=n$.

Let the system and filter equations be given by (11), (21), and (3), and all system parameters not depend on time (i. e. $\Phi_{t i}=\Phi_{i}, H_{t i}=H_{i}, B_{t i}=B_{i}$, $\left.\Gamma_{t i}=\Gamma_{i}\right)$.

Suppose that in filter equations we use system parameters $\Phi_{1}, H_{1}, B_{1}, \Gamma_{1}$ corresponding to the change in the characteristics (i.e. hypothesis $\mathcal{H}_{1}$ ) instead of true values of matrix parameters $\Phi_{0}, H_{0}, B_{0}, \Gamma_{0}$ (i.e. hypothesis $\mathcal{H}_{0}$ ). In this case, in order to determine the values of $\mu_{0}$ and $\mu_{1}$, we need to know the actual correlation matrix of residuals $\bar{\Sigma}_{t 0}=E\left\{\nu_{t 0} \nu_{t 0}^{T}\right\}$. In the case of optimal Kalman filter, the residuals $\nu(t)$ have zero mean and covariance matrix $\Sigma_{t 0}$.

Let us write $\bar{\Sigma}_{t 0}$ as

$$
\bar{\Sigma}_{t 0}=E\left\{\nu_{t 0} \nu_{t 0}^{T}\right\}=E\left\{\left(H_{0} x_{t}-H_{1} \hat{x}_{t}^{-}\right)\left(H_{0} x_{t}^{T}-H_{1}\left(\hat{x}_{t}^{-}\right)^{T}\right)\right\}+I .
$$

After the expansion, we obtain the formula for calculation of correlation matrix of residuals

$$
\begin{aligned}
\bar{\Sigma}_{t 0}= & H_{0} E\left\{x_{t} x_{t}^{T}\right\} H_{0}^{T}-H_{0} E\left\{x_{t}\left(\hat{x}_{t}^{-}\right)^{T}\right\} H_{1}^{T} \\
& +H_{1} E\left\{\hat{x}_{t}^{-}\left(\hat{x}_{t}^{-}\right)^{T}\right\} H_{1}^{T}-H_{1} E\left\{\hat{x}_{t}^{-} x_{t}^{T}\right\} H_{0}^{T}+I .
\end{aligned}
$$

For its calculation, we need to first solve the following difference equations:

$$
\begin{aligned}
E\left\{x_{t} x_{t}^{T}\right\}= & \Phi_{0} E\left\{x_{t-1} x_{t-1}^{T}\right\} \Phi_{0}^{T}+\Gamma_{0} \Gamma_{0}^{T}+B_{0} u_{t-1} E\left\{x_{t-1}^{T}\right\} \Phi_{0}^{T} \\
& +\Phi_{0} E\left\{x_{t-1}\right\} u_{t-1}^{T} B_{0}^{T}+B_{0} u_{t-1} u_{t-1}^{T} B_{0}^{T} \\
E\left\{x_{t}\left(\hat{x}_{t}^{-}\right)^{T}\right\}= & \Phi_{0} E\left\{x_{t-1}\left(\hat{x}_{t-1}^{+}\right)^{T}\right\} \Phi_{1}^{T}+\Phi_{0} E\left\{x_{t-1}\right\} u_{t-1}^{T} B_{1}^{T}
\end{aligned}
$$




$$
\begin{aligned}
& +B_{0} u_{t-1} E\left\{\left(\hat{x}_{t-1}\right)^{T}\right\} \Phi_{1}^{T}+B_{0} u_{t-1} u_{t-1}^{T} B_{1}^{T} \\
E\left\{x_{t}\left(\hat{x}_{t}^{+}\right)^{T}\right\}= & E\left\{x_{t}\left(\hat{x}_{t}^{-}\right)^{T}\right\}\left(I-H_{1}^{T} K_{t}\right)+E\left\{x_{t} x_{t}^{T}\right\} H_{0}^{T} K_{t} \\
E\left\{\hat{x}_{t}^{-}\left(\hat{x}_{t}^{-}\right)^{T}\right\}= & \Phi_{1} E\left\{\hat{x}_{t-1}^{+}\left(\hat{x}_{t-1}^{+}\right)^{T}\right\} \Phi_{1}^{T}+\Phi_{1} E\left\{\hat{x}_{t-1}^{+}\right\} u_{t-1}^{T} B_{1}^{T} \\
& +B_{1} u_{t-1} E\left\{\left(\hat{x}_{t-1}^{+}\right)^{T}\right\} \Phi_{1}^{T}+B_{1} u_{t-1} u_{t-1}^{T} B_{1}^{T} \\
E\left\{\hat{x}_{t}^{+}\left(\hat{x}_{t}^{+}\right)^{T}\right\}= & \left(I-K_{t} H_{1}\right) E\left\{\hat{x}_{t}^{-}\left(\hat{x}_{t}^{-}\right)^{T}\right\}\left(I-H_{1}^{T} K_{t}^{T}\right) \\
& +\left(I-K_{t} H_{1}\right) E\left\{\hat{x}_{t}^{-} x_{t}^{T}\right\} H_{0}^{T} K_{t}^{T} \\
& +K_{t} H_{0} E\left\{x_{t}\left(\hat{x}_{t}^{-}\right)^{T}\right\}\left(I-H_{1}^{T} K_{1}^{T}\right) \\
& +K_{t} H_{0} E\left\{x_{t} x_{t}^{T}\right\} H_{0}^{T} K_{t}^{T}+K_{t} K_{t}^{T} \\
E\left\{\hat{x}_{t}^{+}\right\}= & \left(I-K_{t} H_{1}\right) E\left\{\hat{x}_{t}^{-}\right\}+K_{t} H_{0} E\left\{x_{t}\right\} \\
E\left\{\hat{x}_{t}^{-}\right\}= & \Phi_{1} E\left\{\hat{x}_{t-1}^{+}\right\}+B_{1} u_{t-1} \\
E\left\{x_{t}\right\}= & \Phi_{0} E\left\{x_{t-1}\right\}+B_{0} u_{t-1}
\end{aligned}
$$

satisfying the initial conditions

$$
\begin{aligned}
E\left\{x_{0} x_{0}^{T}\right\} & =E\left\{x_{0}\left(\hat{x}_{0}^{+}\right)^{T}\right\}=E\left\{\hat{x}_{0}^{+}\left(\hat{x}_{0}^{+}\right)^{T}\right\}=P_{0}+\bar{x}_{0} \bar{x}_{0}^{T} \\
E\left\{x_{0}\right\} & =E\left\{\hat{x}_{0}^{+}\right\}=\bar{x}_{0} .
\end{aligned}
$$

Similarly, we can obtain the formula for $\bar{\Sigma}_{t 1}$ when true values of matrix parameters are $\Phi_{1}, H_{1}, B_{1}, \Gamma_{1}$ (this corresponds to hypothesis $\mathcal{H}_{1}$ ), but in filter equations parameters $\Phi_{0}, H_{0}, B_{0}, \Gamma_{0}$ are used.

Consider the equilibrium solutions

$$
\begin{aligned}
& \Sigma_{0}=\lim _{t \rightarrow \infty} \Sigma_{t 0}, \quad \Sigma_{t 0}^{-1}=\left[\sigma_{i j}^{0}\right] \\
& \Sigma_{1}=\lim _{t \rightarrow \infty} \Sigma_{t 1}, \quad \Sigma_{t 1}^{-1}=\left[\sigma_{i j}^{1}\right] \\
& \bar{\Sigma}_{0}=\lim _{t \rightarrow \infty} \bar{\Sigma}_{t 0}=\left[\bar{\sigma}_{i j}^{0}\right], \quad \bar{\Sigma}_{1}=\lim _{t \rightarrow \infty} \bar{\Sigma}_{t 1}=\left[\bar{\sigma}_{i j}^{1}\right] \text {. }
\end{aligned}
$$

Then

$$
\begin{aligned}
& 2 \mu_{1}=\log \operatorname{det} \Sigma_{0}-\log \operatorname{det} \Sigma_{1}+\sum_{i, j=1}^{m}\left(\bar{\sigma}_{i j}^{0} \sigma_{i j}^{0}\right)-m \\
& 2 \mu_{0}=\log \operatorname{det} \Sigma_{0}-\log \operatorname{det} \Sigma_{1}-\sum_{i, j=1}^{m}\left(\bar{\sigma}_{i j}^{1} \sigma_{i j}^{1}\right)+m .
\end{aligned}
$$

Now we can find the average sample numbers:

$\bar{N}_{B}$ is the average number of samples required to reach any threshold from the start of the test, assuming $\mathcal{H}_{0}$ is true;

$\bar{N}_{A}$ is the average number of samples required to reach any threshold from the start of the test, assuming $\mathcal{H}_{1}$ is true.

These sample numbers are given by

$$
\begin{aligned}
& \bar{N}_{B}=[\alpha A+(1-\alpha) B] / \mu_{0} \\
& \bar{N}_{A}=[(1-\beta) A+\beta B] / \mu_{1} .
\end{aligned}
$$


Let $M$ be the necessary number of samples for decision making in the fault point detection problem. We evaluate $M$ as

$$
M=\max \left(\bar{N}_{A}, \bar{N}_{B}\right) .
$$

That means that on the testing interval $\left[t_{0}, t_{N}\right]$ at each time moment only $M+1$ Kalman filters can work simultaneously, and each Kalman filter is based on the corresponding hypothesis.

From the beginning of the test through the first $M$ steps, filters $\mathcal{F}_{i}, i=$ $1, \ldots, M$, enter the Bank. They match the Filter $\mathcal{F}_{0}$ corresponding the hypothesis $\mathcal{H}_{0}$ which means that "the fault point was not detected on the $M$ steps of the test".

The ratio test for system fault point detection is the same as (8).

The only difference is that competitive filters make a queue, moreover each filter works for only a limited number of steps (in our case, $M$ steps). At the $(M+1)$-th step of the test, the filter $\mathcal{F}_{i}$, which was in the Bank during $M$ steps and has not made the decision (this corresponds to condition $B<\lambda_{1_{i} 0}<A$ ), is excluded from the Bank. Instead, the new filter $\mathcal{F}_{M+1}$ is based on the hypothesis $\mathcal{H}_{M+1}$ which means that "the system fault occurred on the $(M+1)$-th step of the test", that is, immediately after moment $t=t_{0}+M+1$.

This algorithm allows us to solve the fault point detection problem with less computational resources consumption.

However, such algorithm is more complex due to the necessity of obtaining the value $M$. This requires us to solve equations (9)-(19). Moreover, if estimate of $M$ is found incorrectly, and it is less than the real number of samples required for decision making, then according to the suggested algorithm the solution may not be found.

\section{Numerical Example}

To show how the algorithms of the previous sections may be used to detect the parameter change point for linear dynamical systems, we consider the following example taken from the inertial navigation [7]:

$$
\begin{aligned}
x_{t+1} & =\left[\begin{array}{lllll}
0.75 & -1.74 & -0.3 & 0.0 & -0.15 \\
0.09 & 0.91 & -0.0005 & 0.0 & -0.008 \\
0.0 & 0.0 & 0.95 & 0.0 & 0.0 \\
0.0 & 0.0 & 0.0 & 0.55 & 0.0 \\
0.0 & 0.0 & 0.0 & 0.0 & 0.905
\end{array}\right] x_{t}+\left[\begin{array}{lll}
0.0 & 0.0 & 0.0 \\
0.0 & 0.0 & 0.0 \\
24.64 & 0.0 & 0.0 \\
0.0 & 0.835 & 0.0 \\
0.0 & 0.0 & 1.83
\end{array}\right] w_{t} \\
z_{t} & =\left[\begin{array}{lllll}
1-e & 0 & 0 & 0 & 1-f \\
0 & 1-g & 0 & 1-h & 0
\end{array}\right] x_{t}+v_{t}, \quad e, f, g, h=\{0,1\}
\end{aligned}
$$

$\left\{w_{t}\right\}$ and $\left\{v_{t}\right\}$ are zero-mean white Gaussian sequences with covariances $Q_{t}=I_{3}$, and $R_{t}=I_{2}$ ( $I_{n}$ is the $n$-dimensional identity matrix). These equations describe the damped Shuler loop driven by the exponentially correlated 3-dimensional noise $w_{t}$ [7]. 
The program for numerical experiments was written in Pascal.

Let us describe the numerical experiments for the problem of fault point detection. Consider the previous example and two hypotheses: $\mathcal{H}_{0}$ which means the nominal mode of the system (the system parameters are $\Phi_{0}=\Phi\left(t_{i}\right), \Gamma_{0}=$ $\Gamma\left(t_{i}\right), B_{0}=B\left(t_{i}\right), H_{0}=H\left(t_{i}\right)$, where $[e, f, g, h]=[0,0,0,0]$; and $\mathcal{H}_{1}$ which means the fault mode of the system (the system parameters are $\Phi_{1}=\Phi\left(t_{i}\right), \Gamma_{1}=$ $\Gamma\left(t_{i}\right), B_{1}=B\left(t_{i}\right), H_{1}=H\left(t_{i}\right)$, where $\{[e, f, g, h] \mid e, f, g, h \in\{0,1\}\} \backslash\{[0,0,0,0]\}$. So, there are 16 types of system faults. All experiments are conducted, provided that the system fault point is random.

Table 1. Detection of the fault point in the system. The type of possible fault is $[0,0,1,0]$. The algorithm with the increasing KFB size.

\begin{tabular}{|l|l|l|l|l|l|l|}
\hline $\begin{array}{l}\text { Experiment } \\
\text { number }\end{array}$ & $\begin{array}{l}\text { LF at the moment } \\
\text { of detection }\end{array}$ & $\begin{array}{l}\text { The fault } \\
\text { point }\end{array}$ & $\begin{array}{l}\text { Detected fault } \\
\text { point }\end{array}$ & $\begin{array}{l}\text { Delay in } \\
\text { detection }\end{array}$ & $\begin{array}{l}\text { Accepted } \\
\text { hypothesis }\end{array}$ & $\begin{array}{l}\text { KFB } \\
\text { size }\end{array}$ \\
\hline 1 & 16.715688 & 39 & 39 & 0 & $\mathcal{H}_{1}$ & 44 \\
2 & 12.159357 & 21 & 22 & 1 & $\mathcal{H}_{1}$ & 22 \\
3 & 66.411028 & 36 & 36 & 0 & $\mathcal{H}_{1}$ & 36 \\
4 & 12.822501 & 33 & 33 & 0 & $\mathcal{H}_{1}$ & 35 \\
5 & 22.600478 & 11 & 25 & 14 & $\mathcal{H}_{1}$ & 26 \\
6 & 96.973967 & 41 & 41 & 0 & $\mathcal{H}_{1}$ & 41 \\
7 & 67.016929
\end{tabular}

Let us demonstrate the efficiency of fault point detection algorithms considered in Sect. 3.1 and Sect. 3.2.

We conducted a series of experiments for the problem of fault point detection. The conditions of experiments are: the error probabilities $\alpha=0.00001$, $\beta=0.00001$, two thresholds $A=11.512915$ and $B=-11.512915$; the point of possible system fault is unknown and random for each experiment. For all experiments, the system fault really takes place. That means that it is necessary to confirm the hypothesis $\mathcal{H}_{1}$ and to detect the fault point by using the available measurements. The experiments are conducted for the faults of two types: $[e, f, g, h]=[0,0,1,0]$ and $[e, f, g, h]=[0,0,0,1]$.

The efficiency of the testing algorithm described in Sect.3.1, can be evaluated according to the data of Table[1. The testing interval here is $[1,50]$, the number of experiments equals 7 , and the series of 500 experiments were also conducted.

According to the experimental data, we conclude that the algorithm with increasing size of the Kalman Filters Bank (KFB) provides a guaranteed solution of the fault point detection problem, but it has one obvious drawback. Since the maximum number of Kalman Filters in the Bank tends to the length of testing interval, the algorithm can not be practically applied to the real life problems.

Now we will try to evaluate the efficiency of the algorithm with the Bank of finite number of competitive Kalman Filters, described in Sect. 3.2 
The results of numerical experiments are shown in Table2 For the realization of a ratio test before testing, we need to evaluate the necessary parameter $M$ (that is the KFB size) by using the equations (9)-(19). It is clear that this estimate will differ in different types of system faults. Table 2 shows the testing results for the system fault $[0,0,1,0]$ and the testing interval $[1,100]$. Given the error probabilities $\alpha$ and $\beta$ and the type of system fault, the maximum number of simultaneously competitive filters needed for the decision making equals 27 .

Table 2. Detection of the fault point in the system. The type of possible fault is $[0,0,1,0]$. The algorithm with the finite KFB.

\begin{tabular}{|l|l|l|l|l|l|l|}
\hline $\begin{array}{l}\text { Experiment } \\
\text { number }\end{array}$ & $\begin{array}{l}\text { LF at the moment } \\
\text { of detection }\end{array}$ & $\begin{array}{l}\text { The fault } \\
\text { point }\end{array}$ & $\begin{array}{l}\text { Detected fault } \\
\text { point }\end{array}$ & $\begin{array}{l}\text { Delay in } \\
\text { detection }\end{array}$ & $\begin{array}{l}\text { Accepted } \\
\text { hypothesis }\end{array}$ & $\begin{array}{l}\text { KFB } \\
\text { size }\end{array}$ \\
\hline 1 & 24.242963 & 48 & 49 & 1 & $\mathcal{H}_{1}$ & 9 \\
2 & 39.596861 & 55 & 55 & 0 & $\mathcal{H}_{1}$ & 24 \\
3 & 18.171810 & 5 & 5 & 0 & $\mathcal{H}_{1}$ & 7 \\
4 & 41.172073 & 40 & 40 & 0 & $\mathcal{H}_{1}$ & 6 \\
5 & 19.113414 & 12 & 17 & 5 & $\mathcal{H}_{1}$ & 27 \\
6 & 15.477792 & 31 & 31 & 0 & $\mathcal{H}_{1}$ & 19 \\
7 & 35.373164 & 11 & 11 & 0 & $\mathcal{H}_{1}$ & 6 \\
8 & 41.409267 & 76 & 77 & 1 & $\mathcal{H}_{1}$ & 12 \\
9 & 18.612180 & 19 & 19 & 0 & $\mathcal{H}_{1}$ & 8 \\
\hline
\end{tabular}

Table 3. Detection of the fault point in the system. The type of possible fault is $[0,0,1,0]$. The algorithm with the finite KFB.

\begin{tabular}{|l|l|l|l|l|l|l|}
\hline $\begin{array}{l}\text { Experiment } \\
\text { number }\end{array}$ & $\begin{array}{l}\text { LF at the moment } \\
\text { of detection }\end{array}$ & $\begin{array}{l}\text { The fault } \\
\text { point }\end{array}$ & $\begin{array}{l}\text { Detected fault } \\
\text { point }\end{array}$ & $\begin{array}{l}\text { Delay in } \\
\text { detection }\end{array}$ & $\begin{array}{l}\text { Accepted } \\
\text { hypothesis }\end{array}$ & $\begin{array}{l}\text { KFB } \\
\text { size }\end{array}$ \\
\hline 1 & 24.242963 & 48 & 49 & 1 & & 9 \\
2 & - & 55 & - & - & - & - \\
3 & 18.171810 & 5 & 5 & 0 & $\mathcal{H}_{1}$ & 7 \\
4 & 41.172073 & 40 & 40 & 0 & $\mathcal{H}_{1}$ & 6 \\
5 & - & 75 & - & - & - & - \\
6 & - & 67 & - & - & - & 6 \\
7 & 35.373164 & 11 & 11 & 0 & $\mathcal{H}_{1}$ & 12 \\
8 & 41.409267 & 76 & 77 & 1 & $\mathcal{H}_{1}$ & 8 \\
9 & 18.612180 & 19 & 19 & 0 & $\mathcal{H}_{1}$ & \\
\hline
\end{tabular}

According to the data in Table 2, the algorithm with the finite number of Kalman filters is efficient for solving the fault point detection problem. So, we have practically verified the correctness of the theoretical estimate for the KFB 
size, because all the experiments required that the real number of competitive filters be less or equal to 27 .

The efficiency of such algorithm essentially depends on the correct choice of the KFB size. If parameter $M$ is incorrect, and is less (but not greater) than the real number of required Kalman filters, then the solution may not be found. Such case is confirmed by the data of Table 3. Here the value of $M$ was chosen to be 10 , but really it must be 27 . According to Table 3, in $50 \%$ of cases the fault point was not detected on all testing interval $[1,100]$ (dash corresponds to these cases).

\section{Conclusion}

The concept of the Bank of Competitive Kalman Filters is applicable to the problem of fault point detection in stochastic system behavior.

The algorithm with increasing number of Kalman filters provides a guaranteed solution to the fault point detection problem, but it has an essential drawback: it can not be practically realized for the real life problems.

To avoid this drawback, another algorithm with the finite size of Kalman Filters Bank was considered in this paper. The authors suggest a method to find the estimate of required size of Kalman Filters Bank. All theoretical results are confirmed by multiple numerical experiments.

\section{References}

1. Newbold, P., M. and Ho Yu-Chi: Detection of Changes in the Characteristics of a Gauss-Markov Process. IEEE Trans. on Aerosp. and Electron. Systems, Vol. AES4(5) (1968) 707-718

2. Hanlon, P., D., Maybeck, P., S.: Equivalent Kalman Filter Bank Structure for Multiple Model Adaptive Estimation (MMAE) and Generalized Likelihood Ratio (GLR) Failure Detection. Proc. of the 36th Conference on Decision \& Control: San Diego California USA 5 (1997) 4312-4317

3. Semoushin, I., V.: The Quickest in the Mean Manoeuvre Detection with the Guaranteed Probability Error (Methods). Shipbuilding: Computing Techniques 26 (1990) 3-7 [In Russian]

4. Semoushin, I., V., Tsyganova, J., V.: An Efficient Way to Evaluate Likelihood Functions in Terms of Kalman Filter Variables. In: Murgu, A. and Lasker, G., E. (eds.): Adaptive, Cooperative and Competitive Processes in Systems Modelling, Design and Analysis. The International Institute for Advanced Studies in Systems Research \& Cybernetics: University of Windsor Windsor Ontario Canada (2001) $67-74$

5. Semoushin, I., V., Tsyganova, J., V., Kulikova, M., V.: On Computation of the Likelihood Ratio Function for Gaussian Markov Signals. In: Andreev, A., C. (ed.): Basic problems of mathematics and mechanics: Ulyanovsk State University Ulyanovsk Russia 2(7) (2000) 93-100 [In Russian]

6. Bierman, G., J.: Factorization Methods for Discrete Sequential Estimation. Academic Press, New-York (1977)

7. Stratton, A.: Combination of Inertial Navigation and Radio Systems. In Borisov, N., I. (ed.): Problems of Inertial Navigation, Mashinostroienie, (1961) [In Russian] 